\title{
SRF accelerators for industrial applications: USPAS Lecture 2021
}

Jayakar "Charles" Thangaraj, Fermilab

Acknowledgment to IARC Fermilab team + several others. 


\section{Accelerators for industry}

- Accelerators for industrial applications:

- Modest energy: few MeVs - tens of MeV

- Modest and high power: tens of kW - hundreds of kW.

- Specific requirements:

- Simplicity

- Low cost

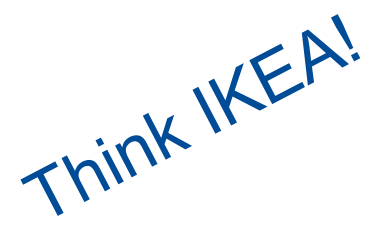

- Reliability

- Work in industrial environment (sometimes harsh)

- Easy to operate

- Small sizes

- High efficiency

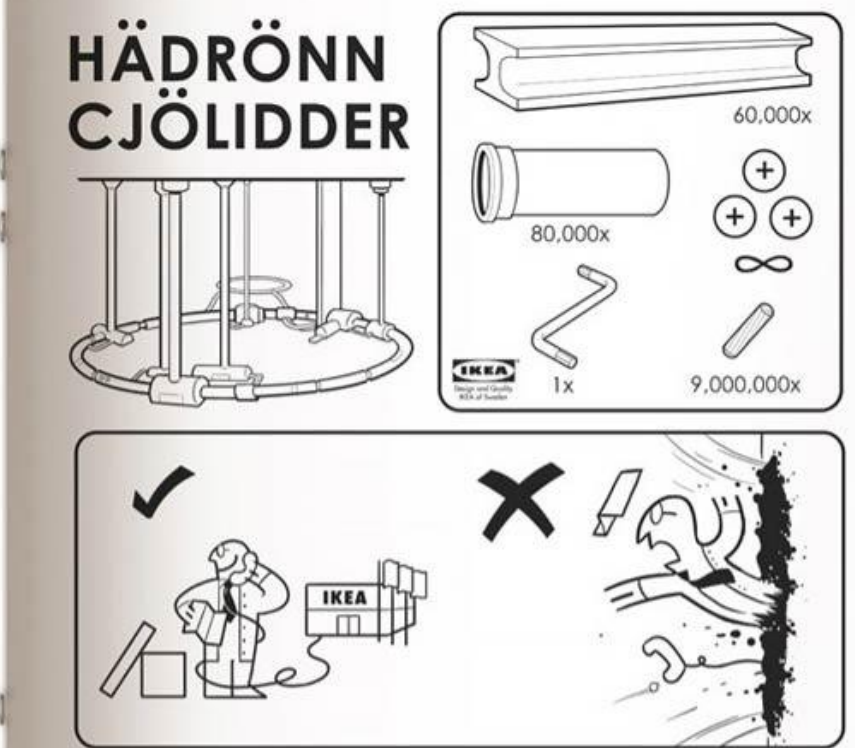

\begin{tabular}{l|l}
\hline & 2.
\end{tabular}
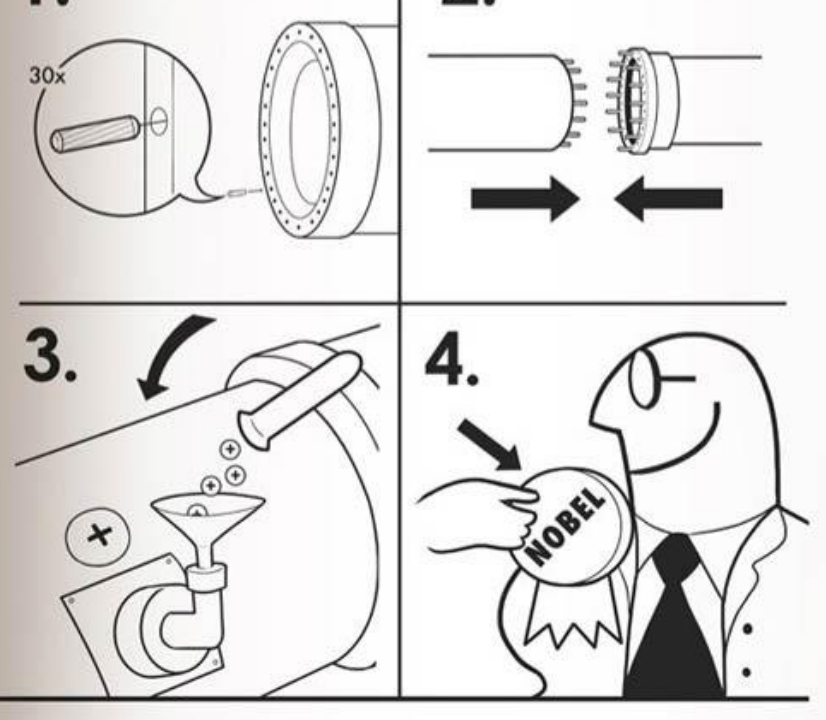


\section{Accelerators come in several sizes and shapes.}

- Electrostatic (few keV - $10 \mathrm{MeV}$ ) - e.g. Dyanmitron, Cockroft-Walton, Pelletron

- Microtron - a cross of cyclotron but uses multi-pass

- Betatron - essentially a transformer but circular can reach several MeV's

- Rhodotron - recirculating through a coaxial cavity

- RF Linac (several MeV's) - normal conducting cavities

- Synchrotron

- Ion accelerators (different species)

A steady market 


\section{Commercial EB accelerator applications are vast}

- EB welding

- EB melting

- EB sterilization

- EB curing

- Non-destructive testing

- Medical imaging

- Cargo inspection

OK....So, if there are accelerators already in market, why do we want SRF technology and what is so attractive with SRF? 


\section{Current vs New Accelerator Technology}

- Bulk materials processing applications require multi-Mev energy for penetration or to generate x-rays and 100's of kW (or even MW) of beam power

- > few MeV accelerators are typically copper and RF driven

- Inherent losses limit efficiency (heat vs beam power) = ops cost

- Heat removal limits duty factor, gradient and average power $\rightarrow$ physically large "fixed" installations = CAPEX

\section{New Technology: Superconducting Radio Frequency (SRF)}

- High wall plug power efficiency (e.g. 75\%)

- Large fraction of the input power goes into beam

- High power \& efficiency enables new \$1 Billion class SRF-based science machines $\rightarrow$ driving large R\&D efforts at labs

- Currently SRF-based science accelerators are huge with complex cryogenic refrigerators, cryomodules, etc. But this is changing!

- Recent SRF breakthroughs now enable a new class of compact, SRF-based industrial accelerators (lower CAPEX and OPS cost) 


\section{Superconducting Radio Frequency (SRF)}

$\sim$ All new high beam power accelerators for discovery science employ SRF

- Why?

- Because all RF power $\rightarrow$ beam power vs heating RF resonators

- SRF $\rightarrow$ Higher gradient, more energy per unit length

- But current SRF "science" accelerators are large and complex

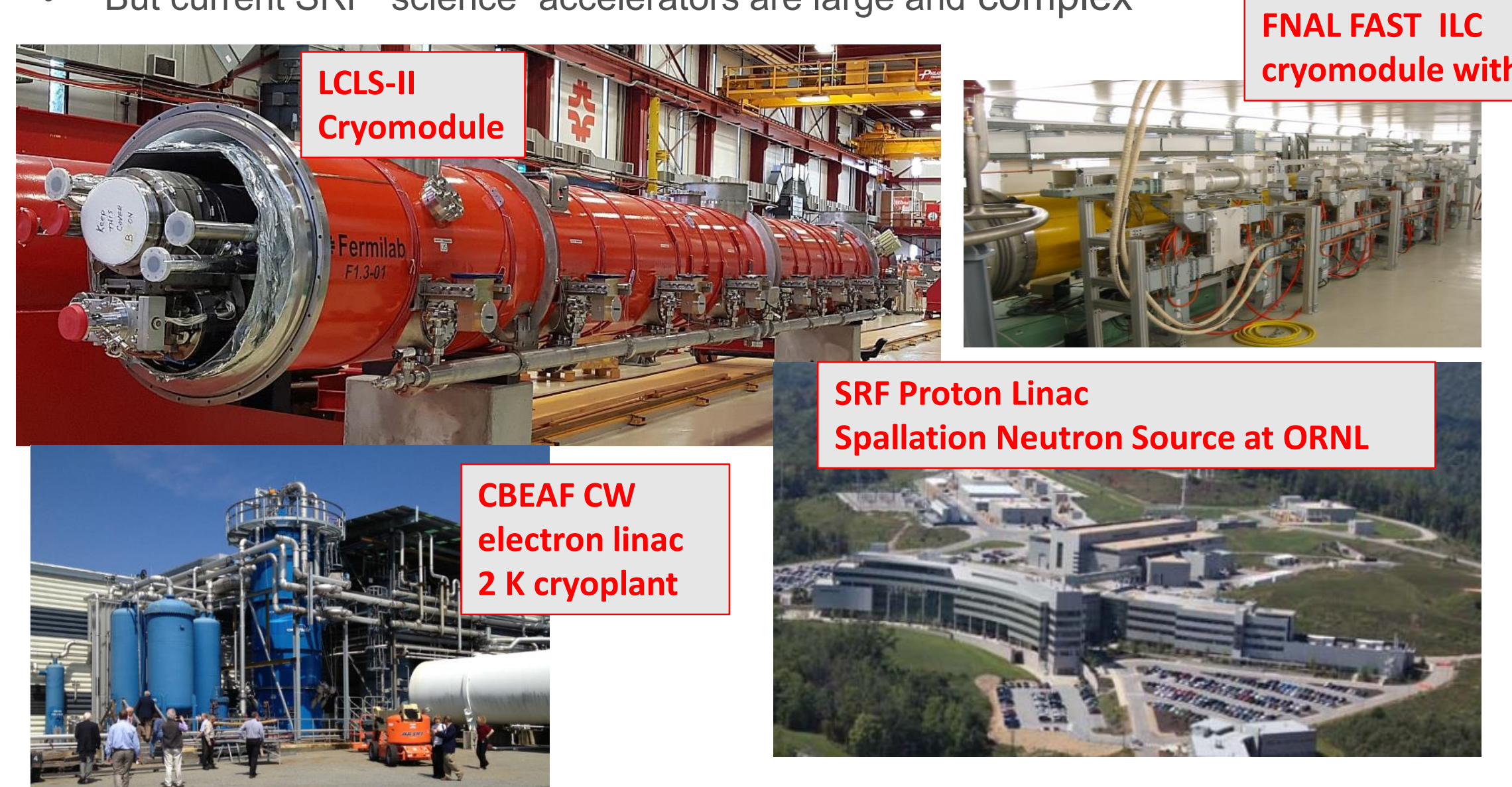


Summary: Superconducting radiofrequency (SRF) technology

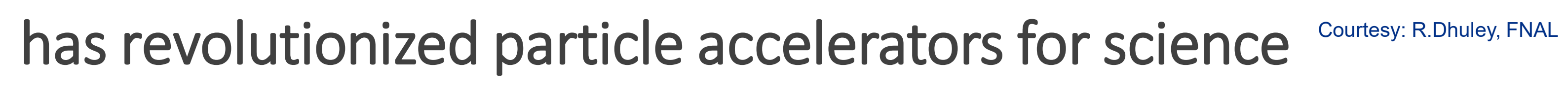
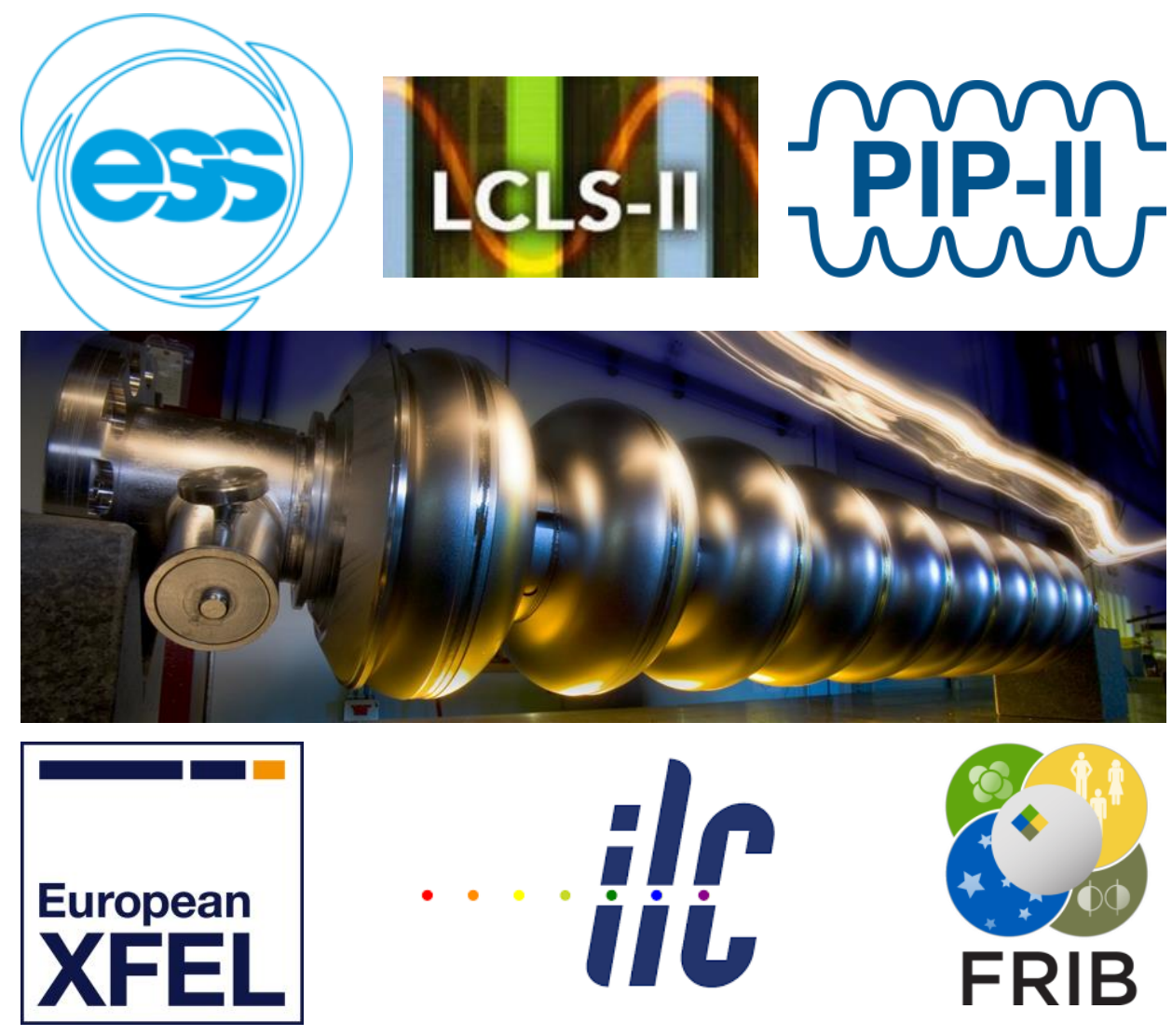

SRF benefits for large scientific machines

$>$ High wall-plug efficiency

$>$ High average beam power

Breakthroughs continue to ensue

$>$ Niobium cavities achieve $>50 \mathrm{MV} / \mathrm{m}$

in $2 \mathrm{~K}$ liquid helium

$>\mathrm{Nb}_{3} \mathrm{Sn}$ cavities attain $>20 \mathrm{MV} / \mathrm{m}$ in

4.5 K liquid helium 


\section{SRF for \\ basic science}

\section{SRF for}

industry \& society

$>$ SRF relevant Industrial applications of particle accelerators?

$>$ How to make SRF suitable for industrial settings? 


\section{Recent SRF Technology Breakthroughs:}

- Higher temperature superconductors: $\mathrm{Nb}_{3} \mathrm{Sn}$ coated cavities dramatically lower cryogenic losses and allow higher operating temperatures (e.g. $4 \mathrm{~K}$ vs $1.8 \mathrm{~K}$ )

- Commercial Cryocoolers: new devices with higher capacity at $4 \mathrm{~K}$ enables turn-key cryogenic systems

- Conduction Cooling: possible with low cavity losses $\rightarrow$ dramatically simplifies cryostats (no Liquid Helium !)

- New RF Power technology: injection locked magnetrons allow phase/amplitude control at high efficiency and much lower cost per watt

- Integrated electron guns: reduce accelerator complexity

- Enable compact industrial SRF accelerators at low cost 


\section{Industrial applications and scope of SRF acceleratồ}

\section{Electron beam radiation processing applications}

Water/sludge/medical waste decontamination

Flue gas cleanup

Medical device sterilization

Strengthening of asphalt pavements Radiation processing requires:

Beam energy: 0.5-10 MeV

Beam power: $>>100 \mathrm{~kW}$

Industrial settings demand:

Low capital and operating expense

Robust, reliable, turnkey operation

http://accelconf.web.cern.ch/AccelConf/napac2016/talks/thb3io02 talk.pdf 1-meter long SRF linac (niobium or $\mathrm{Nb}_{3} \mathrm{Sn}$ cavities)

operating at $10 \mathrm{MV} / \mathrm{m}$ can provide the required energy

Small SRF surface resistance enables continuous wave (cw) operation, leading to high average beam power

At present, SRF accelerators are designed to operate with complex liquid helium cryogenic systems!

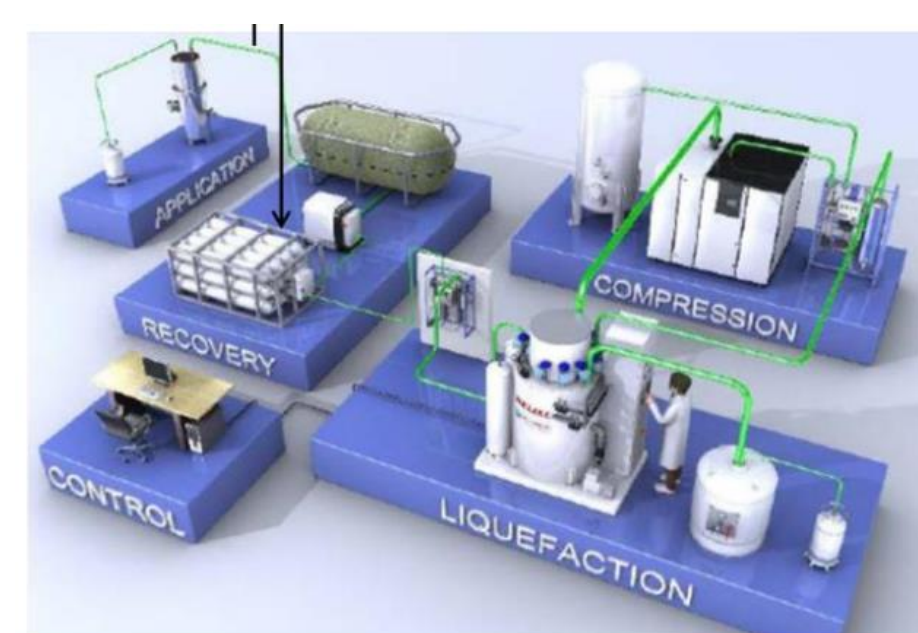

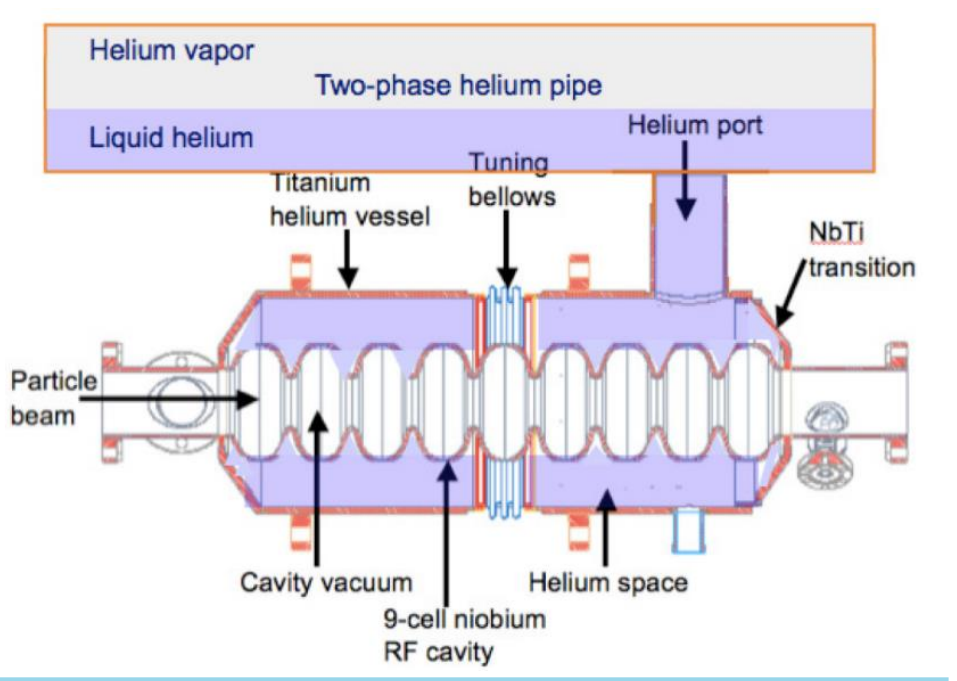




\section{Why superconducting: HIGH Power (CW)}

\begin{tabular}{|c|c|c|c|}
\hline Technology & Energy & Power & Issues/Potential \\
\hline Room temperature & Few MeV & Up to few hundred kW's & $\begin{array}{l}\text { - Energy efficiency } \\
\text { - Heat loss } \\
\text { - Old(er) technology }\end{array}$ \\
\hline Superconducting & $10 \mathrm{MeV}$ & $100 \mathrm{~kW}-1+\mathrm{MW}$ & $\begin{array}{l}\text { - } \text { CW } \\
\text { - Excellent energy efficiency } \\
\text { - } \text { Reliable, cutting-edge } \\
\text { technology based on science } \\
\text { machines }(>1 \$ B) \\
\text { - Compact cryogenics }\end{array}$ \\
\hline
\end{tabular}




\section{Concept of a cryocooler conduction-cooled SRF accelerator}

R.D. Kephart, SRF2015, 2015. https://accelconf.web.cern.ch/srf2015/papers/frba03.pdf Patents: US10390419B2, US10070509B2, US9642239B2

AN ILLUSTRATED EXAMPLE

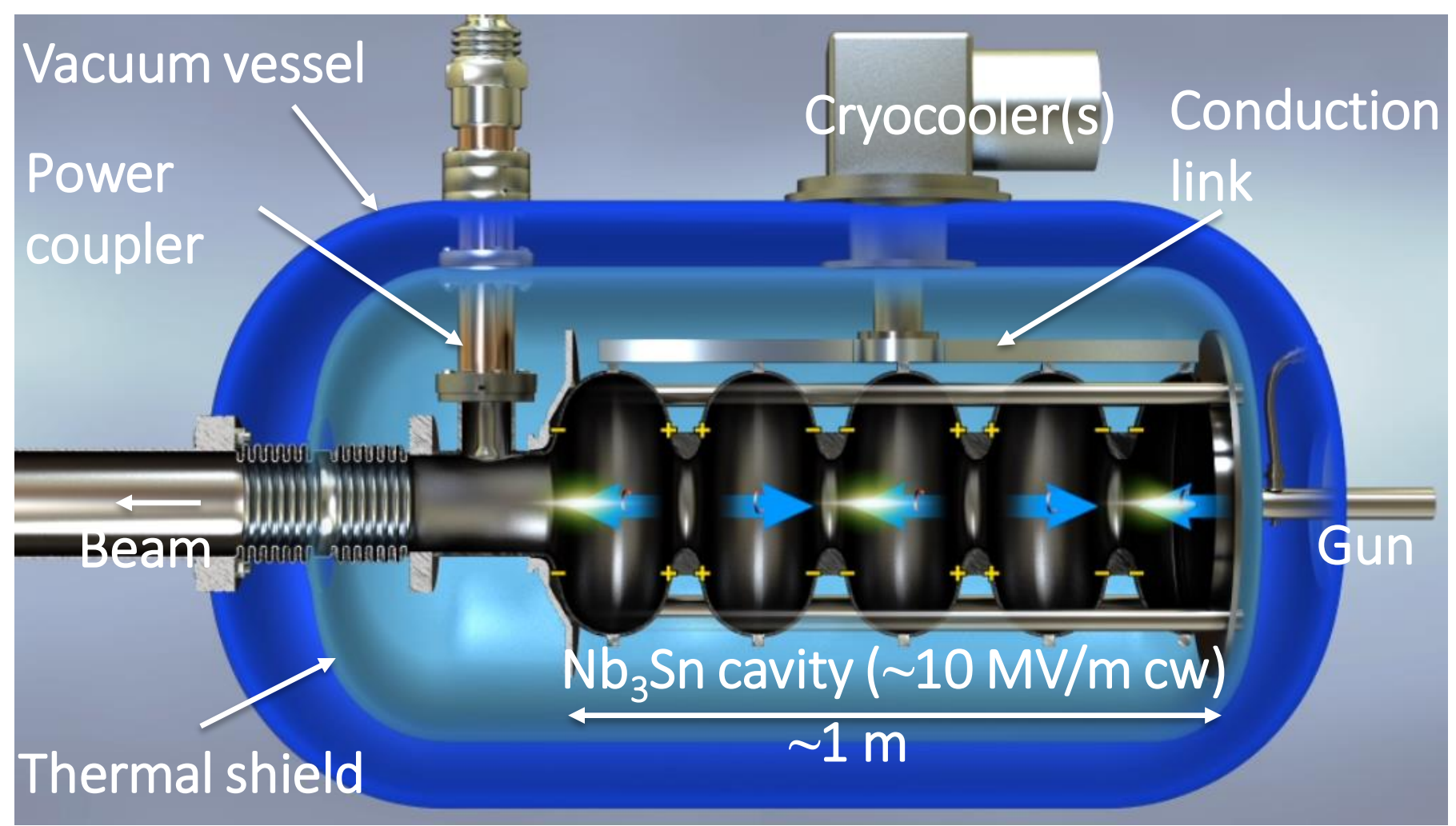

All cryogenics integrated into the module

- Cryocooler $4 \mathrm{~K}$ stage cools the SRF cavity

- Cryocooler $45 \mathrm{~K}$ stage cools thermal shield/intercept

- Enclosed in a simple vacuum vessel 


\section{A new frontier in SRF is simplifying the cooling methods!}

Fermilab

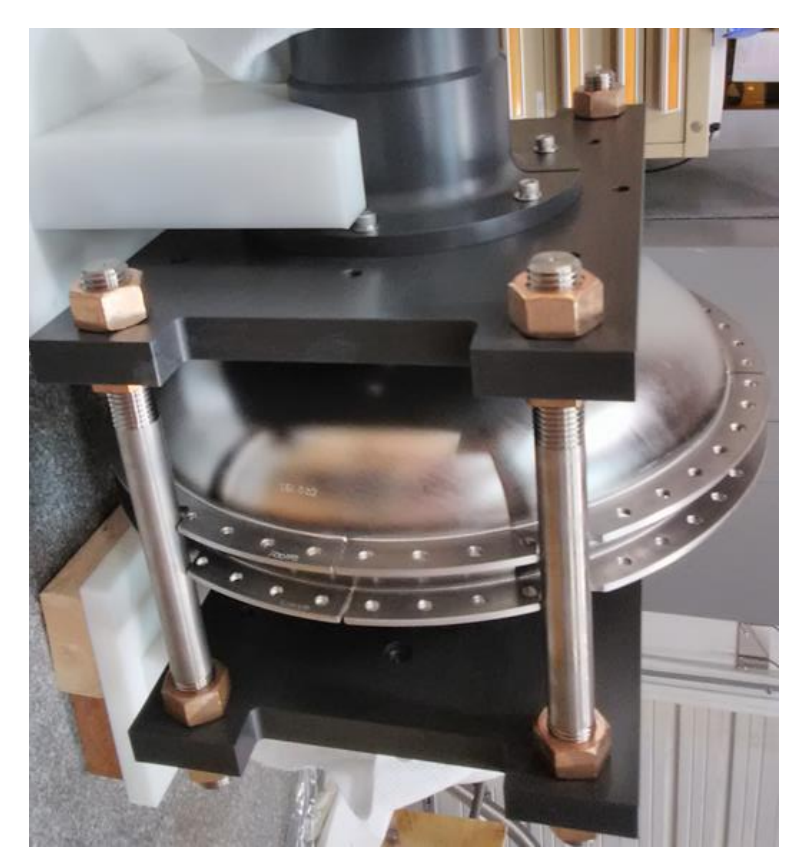

$>650 \mathrm{MHz}$

$>$ welded niobium rings
Jefferson Lab

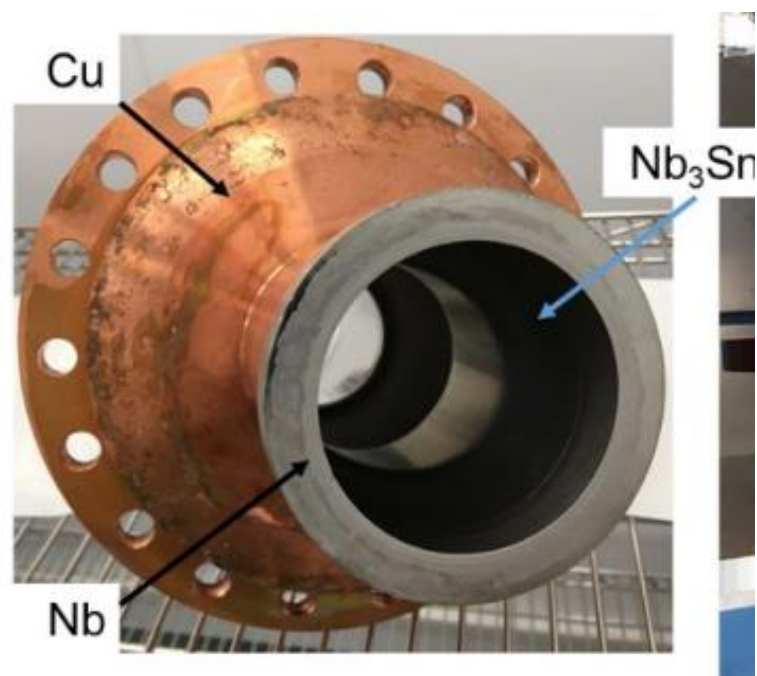

https://doi.org/10.1088/1757-899X/755/1/012136

$>1.5 \mathrm{GHz}$

$>$ Cold sprayed + electrodeposited copper
Cornell University

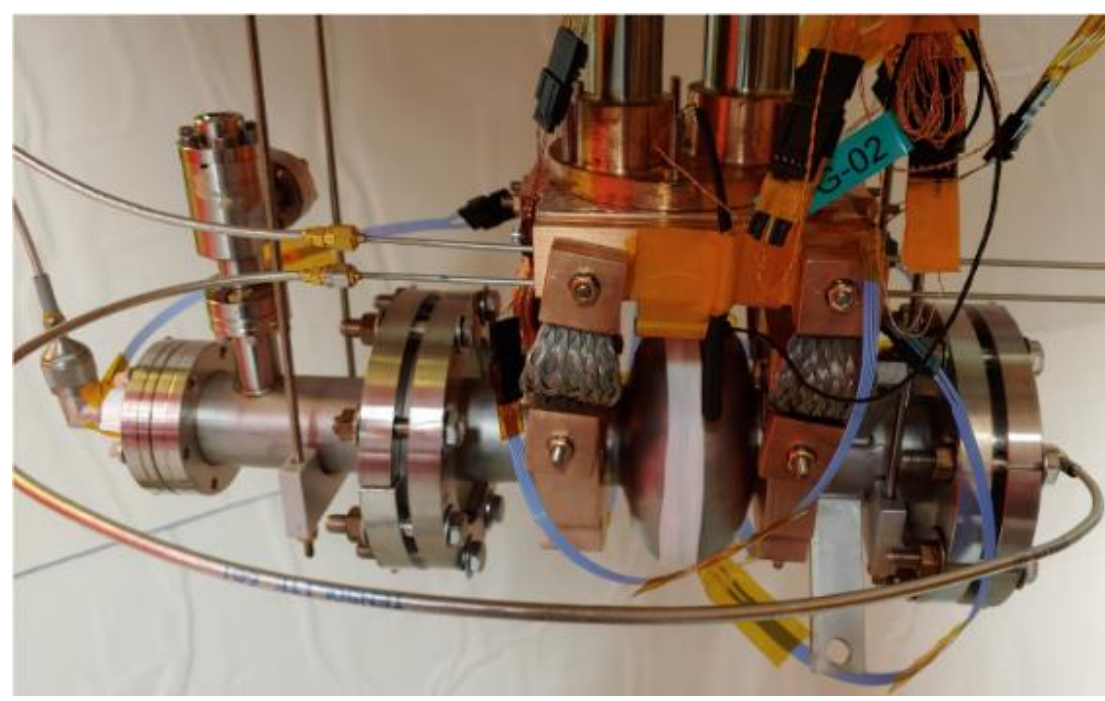

https://arxiv.org/abs/2002.11755

$>2.6 \mathrm{GHz}$

$>$ Copper clamps 


\section{Types of industrial accelerators}

- Dept. of Energy provided funding to develop novel accelerator designs to address need for industrial application in the energy and environment applications

\begin{tabular}{|c|c|c|c|c|}
\hline & $\begin{array}{l}\text { Type } 1 \\
\text { Demo/Small } \\
\text { Scale }\end{array}$ & $\begin{array}{l}\text { Type } 2 \\
\text { Medium } \\
\text { Scale Low } \\
\text { Energy }\end{array}$ & $\begin{array}{l}\text { Type } 3 \\
\text { Medium Scale } \\
\text { High Energy }\end{array}$ & $\begin{array}{l}\text { Type } 4 \\
\text { Large Scale } \\
\text { High Energy }\end{array}$ \\
\hline $\begin{array}{l}\text { Example } \\
\text { Applications }\end{array}$ & $\begin{array}{l}\text { R\&D, } \\
\text { Sterilization, } \\
\text { industrial } \\
\text { effluent streams }\end{array}$ & $\begin{array}{l}\text { Flue Gas, } \\
\text { Waste water }\end{array}$ & $\begin{array}{l}\text { Wastewater, } \\
\text { sludge, medical } \\
\text { waste }\end{array}$ & $\begin{array}{l}\text { Sludge, Medical } \\
\text { waste, Env. } \\
\text { remediation }\end{array}$ \\
\hline $\begin{array}{l}\text { Electron Beam } \\
\text { Energy }\end{array}$ & $0.5-1.5 \mathrm{MeV}$ & $1-2 \mathrm{MeV}$ & $10 \mathrm{MeV}$ & $10 \mathrm{MeV}$ \\
\hline $\begin{array}{l}\text { Electron Beam } \\
\text { Power }(\mathrm{CW})\end{array}$ & $>0.5 \mathrm{MW}$ & $>1 \mathrm{MW}$ & $>1 \mathrm{MW}$ & $>10 \mathrm{MW}$ \\
\hline $\begin{array}{l}\text { Wallplug } \\
\text { Efficiency }\end{array}$ & $>50 \%$ & $>50 \%$ & $>50 \%$ & $>75 \%$ \\
\hline $\begin{array}{l}\text { Target Capital } \\
\text { Cost* }\end{array}$ & $<\$ 10 / \mathrm{W}$ & $<\$ 10 / W$ & $<\$ 10 / W$ & $<\$ 5 / W$ \\
\hline $\begin{array}{l}\text { Target Operating } \\
\text { Cost } \uparrow\end{array}$ & $<1.0 \mathrm{M} \$ / \mathrm{yr}$ & $<1.5 \mathrm{M} \$ / \mathrm{yr}$ & $<1.5 \mathrm{M} \$ / \mathrm{yr}$ & $<12 \mathrm{M} \$ / \mathrm{yr}$ \\
\hline
\end{tabular}


$1 \mathrm{MeV}, 1 \mathrm{MW}$ SRF accelerator

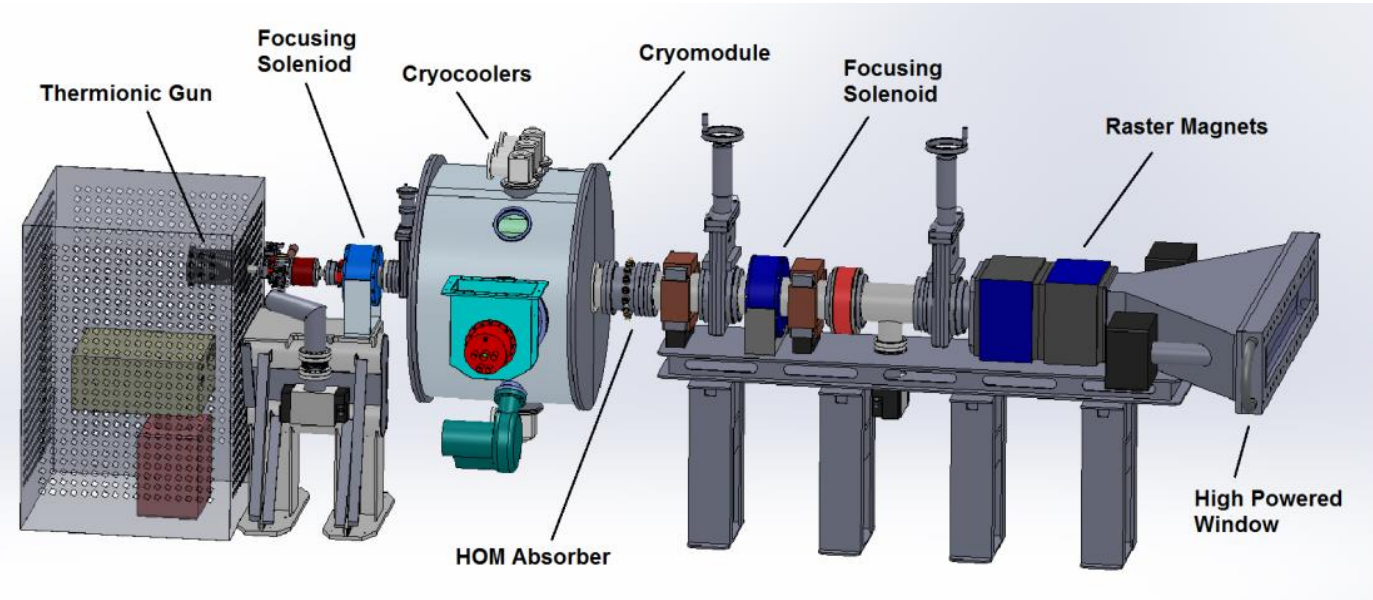

Jefferson Lab

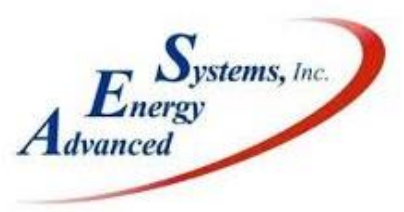

GENERAL ATOMICS
G. Ciovati, R. Rimmer, F. Hannon, J. Guo, F. Marhauser, V. Vylet

J. Rathke, T. Schultheiss

J. Anderson, B. Coriton, L. Holland, M. LeSher

\section{$10 \mathrm{MeV}, 1 \mathrm{MW}$ SRF accelerator}

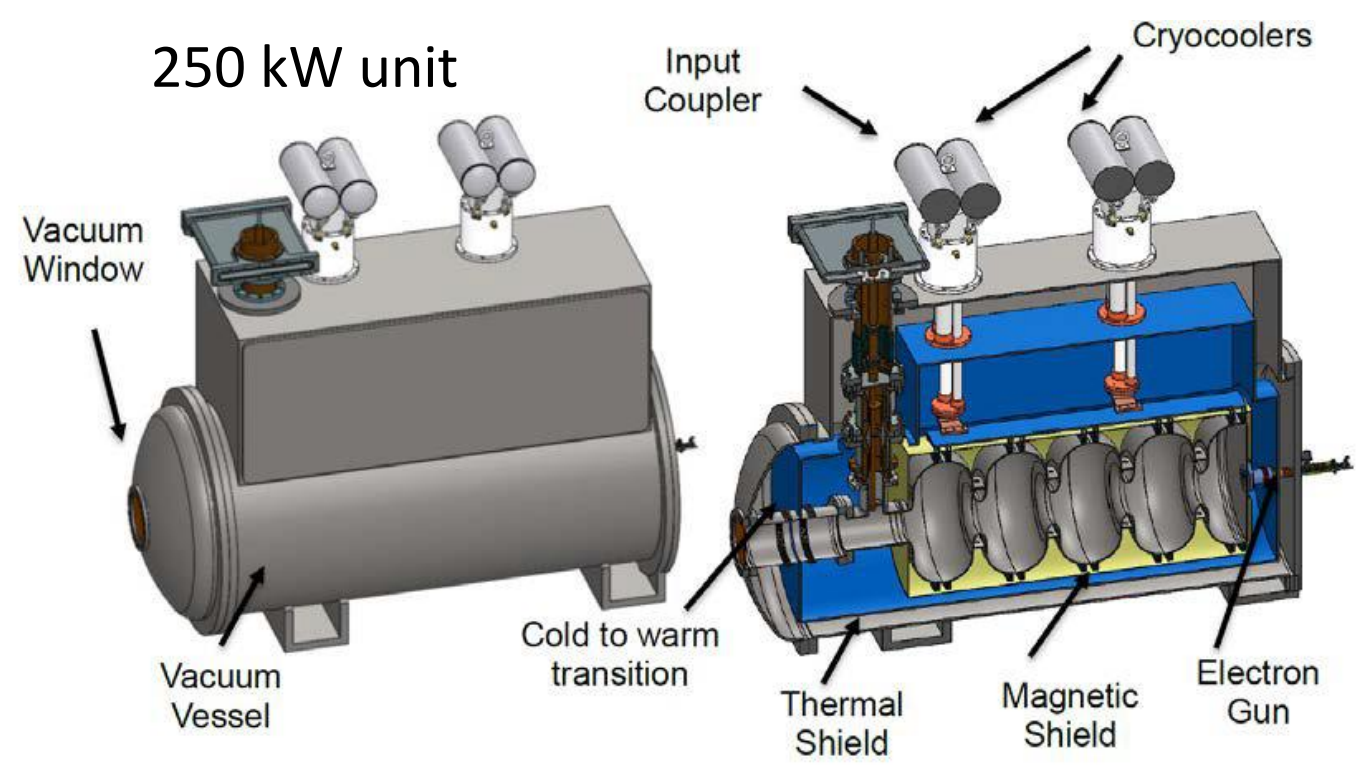

R. Kephart, V. Yakovlev, N. Solyak , I. Gonin , S. Kazakov , 굴 Fermilab T. Khabiboulline, O. Prokofiev, S. Posen

T. Kroc, C. Cooper, J. Thangaraj, R. Dhuley, M. Geelhoed

Northern Illinois Philippe Piot
University

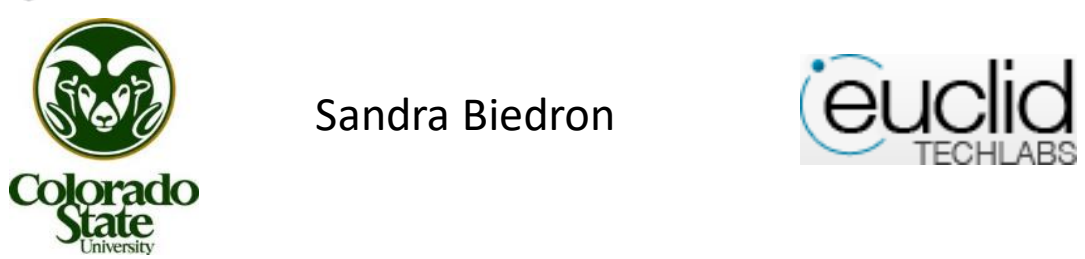

A. Kanareykin 


\section{Design and economics studies of industrial scale SRF electron accelerators ( $10 \mathrm{MeV},>>100 \mathrm{~kW}$ )}

Supported by US Dept. of Energy HEP Accelerator Stewardship Program

\begin{tabular}{|c|c|c|}
\hline $\begin{array}{l}\text { Phase (year) / } \\
\text { Fermilab PI }\end{array}$ & Activity & Stewardship partner \\
\hline $\begin{array}{l}\text { I (2016-17) / } \\
\text { R.D. Kephart }\end{array}$ & $\begin{array}{l}\text { Conceptual design of a } 250 \mathrm{~kW} \text { and economic } \\
\text { analysis of a } 1000 \mathrm{~kW} \text { facility }\end{array}$ & \multirow{2}{*}{$\begin{array}{l}\text { MWRD of } \\
\text { Greater } \\
\text { Chicago }\end{array}$} \\
\hline $\begin{array}{l}\text { II (2017-18) / } \\
\text { J.C.T. Thangaraj }\end{array}$ & $\begin{array}{l}\text { Conceptual design of a } 1000 \mathrm{~kW} \text { module and } \\
\text { economic analysis of a } 10000 \mathrm{~kW} \text { facility }\end{array}$ & \\
\hline $\begin{array}{l}\text { III (2019-in progress) / } \\
\text { R.C. Dhuley }\end{array}$ & $\begin{array}{l}\text { Practical cryogenic design and cost analysis of a } \\
1000 \mathrm{~kW} \text { module (Publication in preparation) }\end{array}$ & \\
\hline
\end{tabular}

Design reports available at: https://iarc.fnal.gov/publications/ 
LETTER • OPEN ACCESS

First demonstration of a cryocooler conduction cooled superconducting radiofrequency cavity operating at practical CW accelerating gradients

R C Dhuley ${ }^{1}$ (D), S Posen ${ }^{1}$ (D), M I Geelhoed ${ }^{1}$, O Prokofiev ${ }^{1}$ and J C T Thangaraj ${ }^{1}$

Published 20 April 2020 • (c) 2020 IOP Publishing Ltd

Superconductor Science and Technology, Volume 33, Number 6

Citation R C Dhuley et al 2020 Supercond. Sci. Technol. 33 06LTO

\section{Article PDF}

Figures - References -

\section{Open Access}

Design of a cw, low-energy, high-power superconducting linac for environmental applications

G. Ciovati, J. Anderson, B. Coriton, J. Guo, F. Hannon, L. Holland, M. LeSher, F. Marhauser, J. Rathke, R. Rimmer, T. Schultheiss, and V. Vylet

Phys. Rev. Accel. Beams 21, 091601 - Published 4 September 2018
773 Total downloads

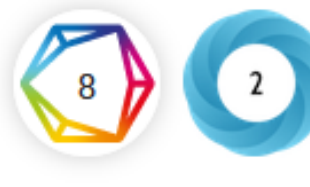

Turn on MathJax

Share this article

田 $\mathbf{f} \mathrm{g}^{+} \mathrm{m}$

\section{Superconductor Science and Technology}

VIEWPOINT • FREE ARTICLE

Towards a cryogen-free practical gradient cW SRF accelerator Andrew J May ${ }^{1,2,3}$ (D)

Published 8 January 2021 • @ 2021 IOP Publishing Ltd

Superconductor Science and Technology Volume 34, Number 2

Citation Andrew J May 2021 Supercond. Sci. Technol. 34020502

\section{article PDF}

References - 


\section{New opportunities (and challenges) with compact industrial SRF- based accelerators}




\section{Future Accelerator Applications}

\section{Energy and Environment}

- Treat Municipal Waste \& Sludge

- Eliminate pathogens in sludge

- Destroy organics, pharmaceuticals in waste water

- In-situ environmental remediation

- Contaminated soils

- Spoils from dredging, etc

\section{Industrial and Security}

- Catalyze Chemical reactions to save time and energy

- In-situ cross-link of materials

- Improve pavement lifetime

- Instant cure coatings

- Medical sterilization without Co60

- Improved non-invasive inspection of cargo containers

These new applications need cost effective, energy efficient, high average power electron beams.

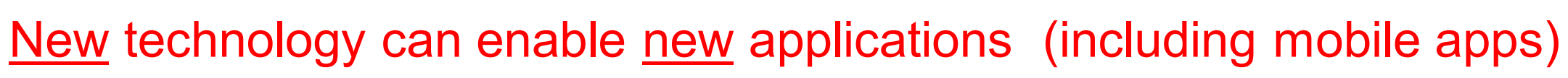




\section{Emerging Application where SRF can play a game changing role}

- Medical device sterilization : A brief background, Co-60, a story!

- "Forever chemicals"

- Pavement application

- Wastewater application . Let me start with this one

Warning: Most of this at R\&"D" SRF Tech and customer/application expectations should be kept in focus

VERY VERY IMP Questions to always ask yourself:

What is the cost economics? What type of cost? CAPEX or OPEX?

What is the business model?

What is the TRL and trends? Where is the cost driver?

What is the customer willing to pay for? Regulatory challenges?

Safety and redundancy for certain application? Portability etc. 


\section{COST DRIVERS: Cost estimate for $1 \mathrm{MeV}, 1 \mathrm{MW}$ SRF EB facility \\ specific application chosen as example)}

\begin{tabular}{|l|l|}
\hline Capital Cost & \\
\hline SRF Accelerator & $\$ 4,500,000$ \\
\hline Infrastructure & $\$ 2,750,000$ \\
\hline Total & $\$ 7,250,000$ \\
\hline Investment (20\%) & $\$ 1,450,000$ \\
\hline Amortization(15yr @ 8\%) & $\$ 670 \mathrm{k} / \mathrm{yr}$ \\
\hline
\end{tabular}

\section{Operating Cost $(8,000 \mathrm{hrs} / \mathrm{yr})$}

\begin{tabular}{l|l} 
Powera) \$159.2/hr &
\end{tabular}

Cooling water None (air-cooled chillers)

Maintenance $^{\text {b) }} \$ 145 \mathrm{k} / \mathrm{yr}$

Total \$1,418,600/yr

$\begin{array}{ll}\text { Total Cost (Capital + Op.) } & \$ 261 / \mathrm{hr} \\ \mathbf{\$ 2 , 0 8 8 , 6 0 0 / \mathrm { yr }}\end{array}$

\section{Assumptions}

a) $2.274 \mathrm{MW}$ (Elec. Eff.: 42\%) @ \$0.07/kWh

b) $2 \%$ capital/year

c) No dedicated operator

QUESTION: Can you see the cost driver?

Electric powe

Amortization

Maintenance 


\section{Processing cost sensitivity...[drives tech development]}
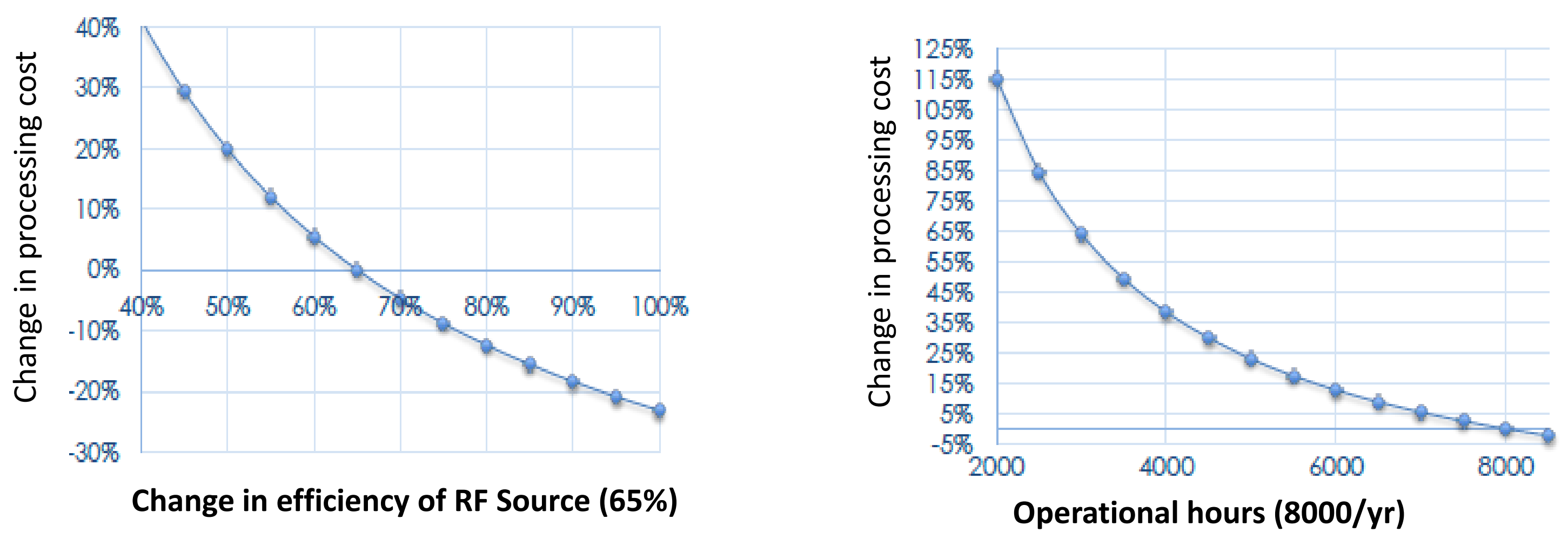

Current technology: klystron (65\%), IOT (70\%)

In development: magnetrons (90\%)

$\mathrm{RF}$ is the driver and we want high reliability to break even quickly 


\section{Processing cost per Application}

\begin{tabular}{|c|c|c|c|}
\hline & \multicolumn{2}{|c|}{$1 \mathrm{MeV}, 1 \mathrm{MW}$} & $10 \mathrm{MeV}, 1 \mathrm{MW}$ \\
\hline & \multicolumn{2}{|c|}{ WASTEWATER } & SLUDGE \\
\hline Dose requirement & $1 \mathrm{kGy}$ & $4 \mathrm{kGy}$ & $10 \mathrm{kGy}$ \\
\hline Processing cost & $\$ 0.13 / \mathrm{m}^{3}$ (\$0.482/kgal) & $\$ 0.51 / \mathrm{m}^{3}$ (\$1.93/kgal) & $\$ 19.7 /$ dry ton \\
\hline $\begin{array}{l}\text { Cost of current technologies } \\
\text { (other than EB) [4] }\end{array}$ & \multicolumn{2}{|c|}{$\$ 0.25 / \mathrm{m}^{3}-\$ 1.00 / \mathrm{m}^{3}$} & $>\$ 50 /$ dry ton \\
\hline Daily Processed Volume & $\begin{array}{l}45,000 \mathrm{~m}^{3} \\
\text { (11.9 Mgal) }\end{array}$ & $\begin{array}{l}11,250 \mathrm{~m}^{3} \\
\text { (3.0 Mgal) }\end{array}$ & $\begin{array}{l}278 \text { dry ton } 112 \mathrm{M} \text { a } \\
\text { with } 25 \% \text { biosolid } \\
\text { waste) }\end{array}$ \\
\hline Required Flow Rate (gpm) & 9,050 & 2,260 & 984 \\
\hline Comments [4] & $\begin{array}{l}\text { Color, Odor, Coliform } \\
\text { bacteria removal }\end{array}$ & Kill $>99 \%$ of bacteria & $\begin{array}{l}\text { Inactivate some } \\
\text { radiation resistant } \\
\text { organisms }\end{array}$ \\
\hline
\end{tabular}




\section{Development of SRF based field emission sources}

\section{PI: Dr. Philippe Piot (NIU/Argonne National Lab.)}

\section{NIU-Fermilab collaboration}

- field emission cathode with nanostructured surface located in high e-field region of an SRF cavity

- use cw operation to produce high repetition rate field emission (high $I_{\text {avg }}$ )

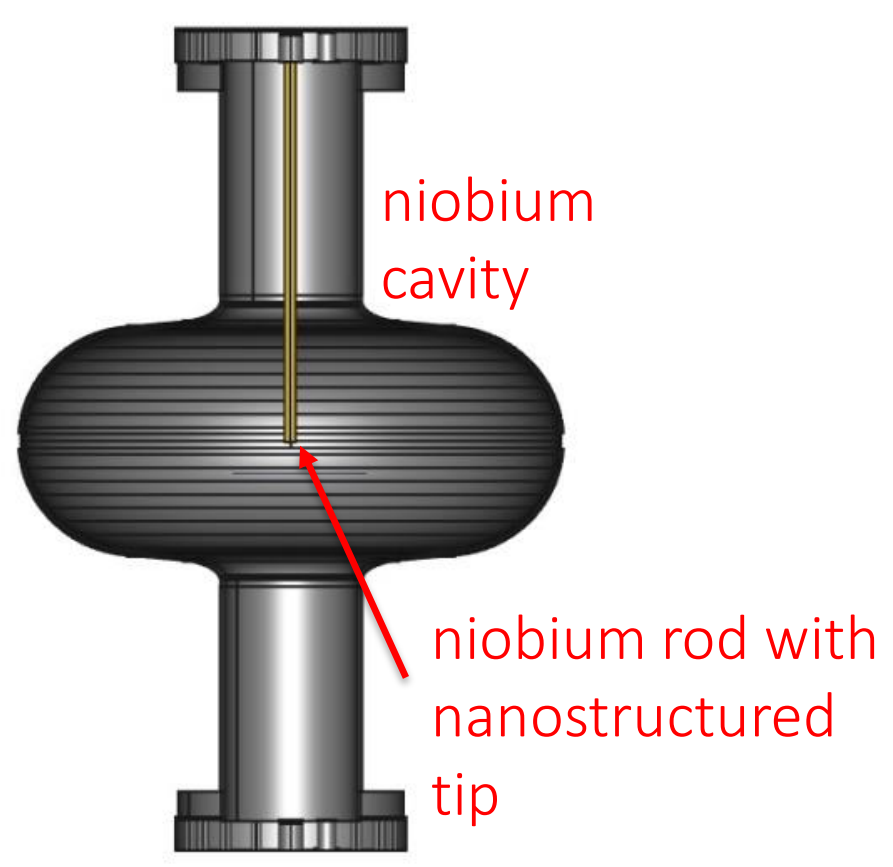

Cathode surface e-field

(650 MHz cavity, 1.6 W cryo-cooling)

Monsen et al., http://accelconf.web.cern.ch/ipac2019/papers/tupts083.pdf
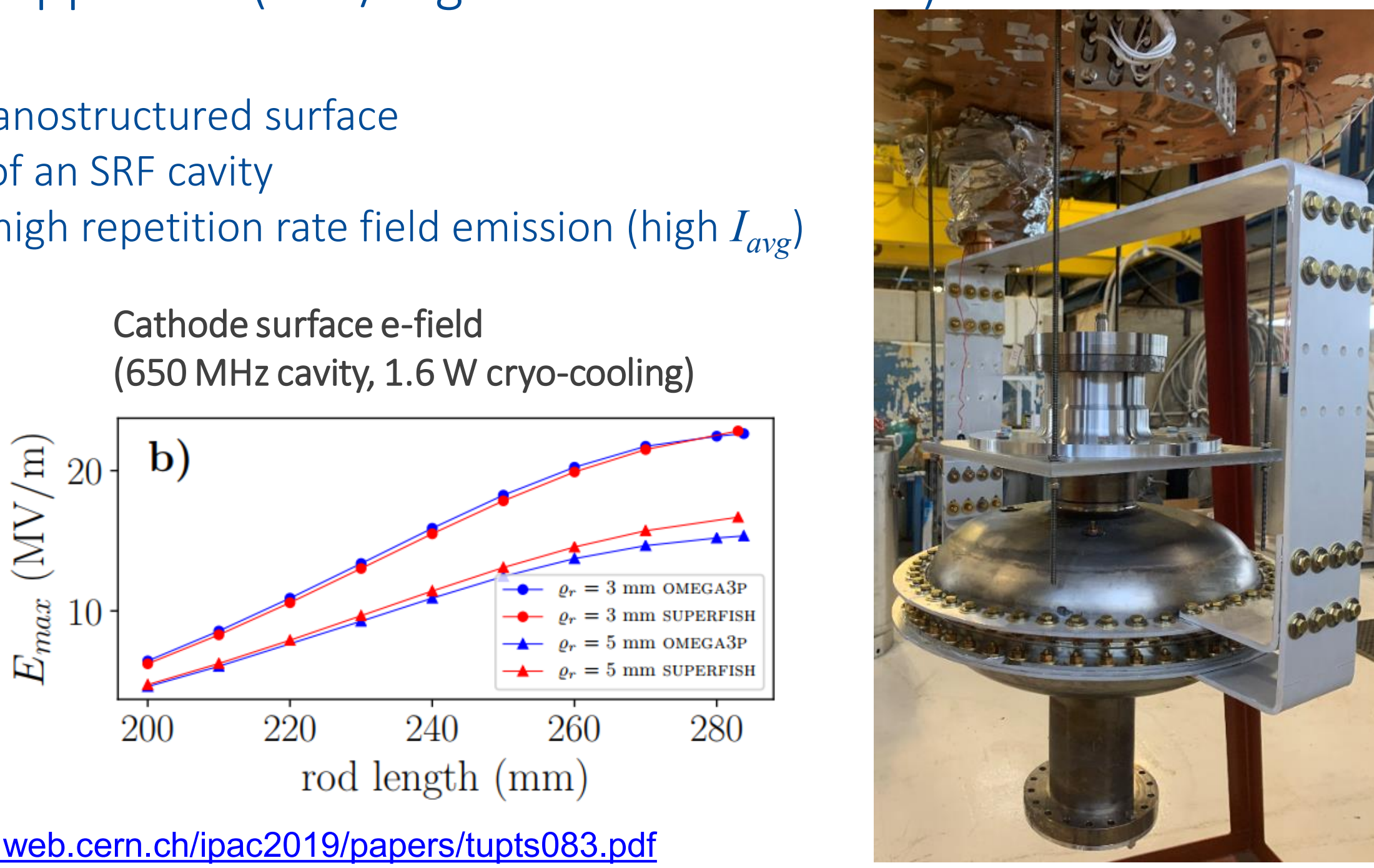


\section{Cryocooled based standalone SRF modules}

Cryocooled SRF has already been picked up by the particle accelerator industry!

S. Kutsaev et al., https://ieeexplore.ieee.org/document/9119112/

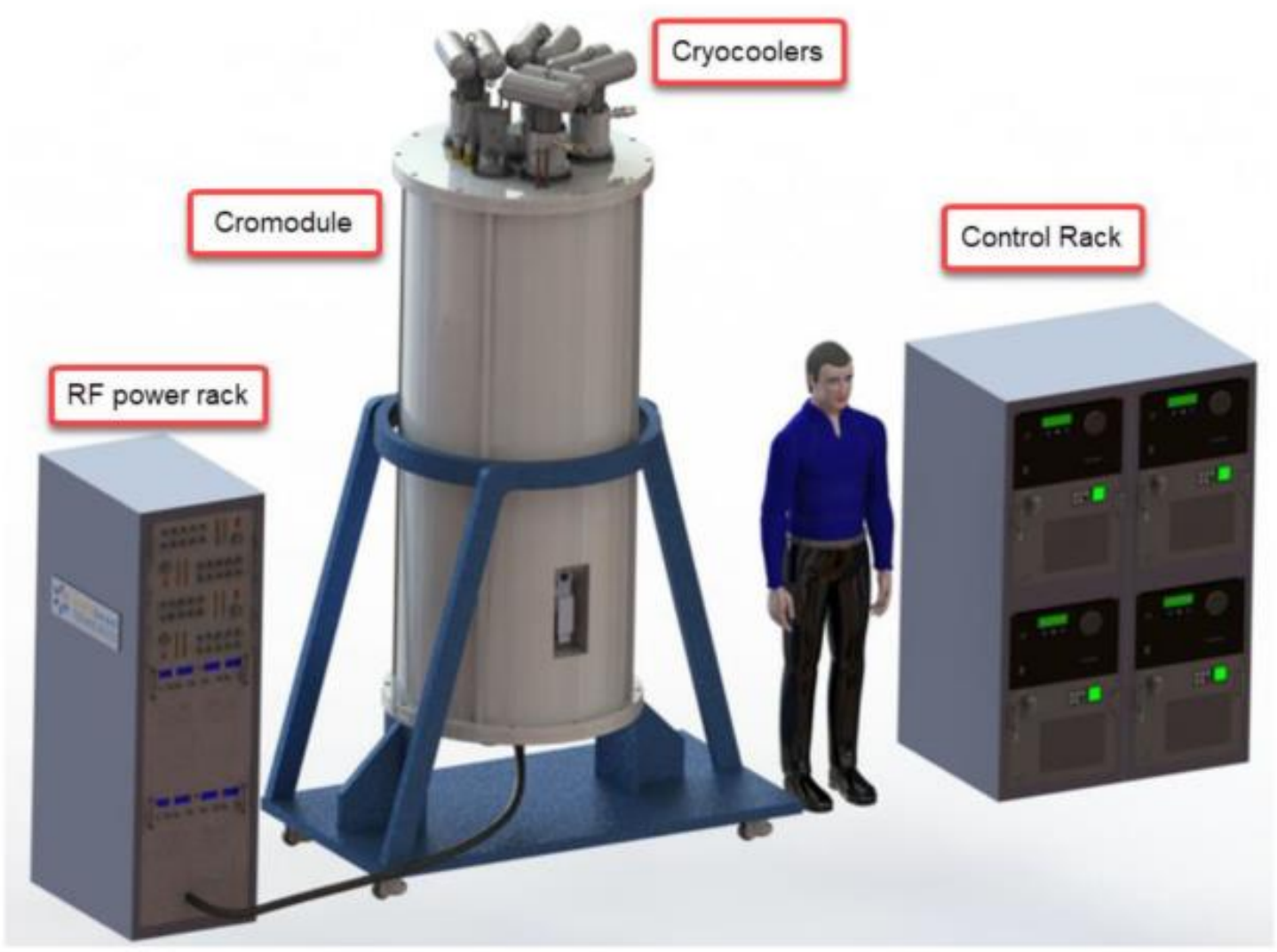

A SRF QWR cooled by pulse tube coolers for beamline upgrade at Argonne Natl. Lab.

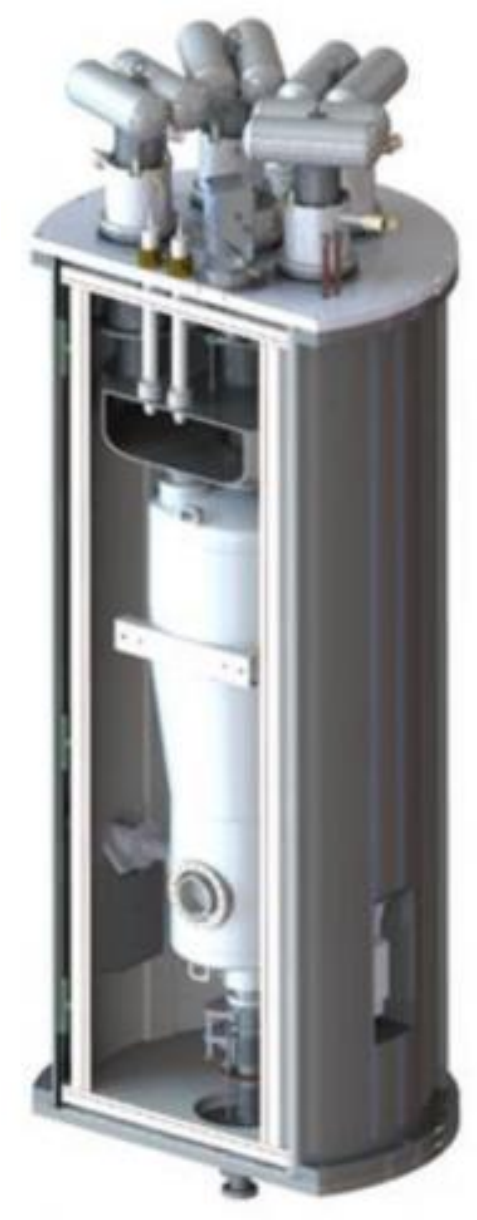




\section{Medical device sterilization: A major application.}
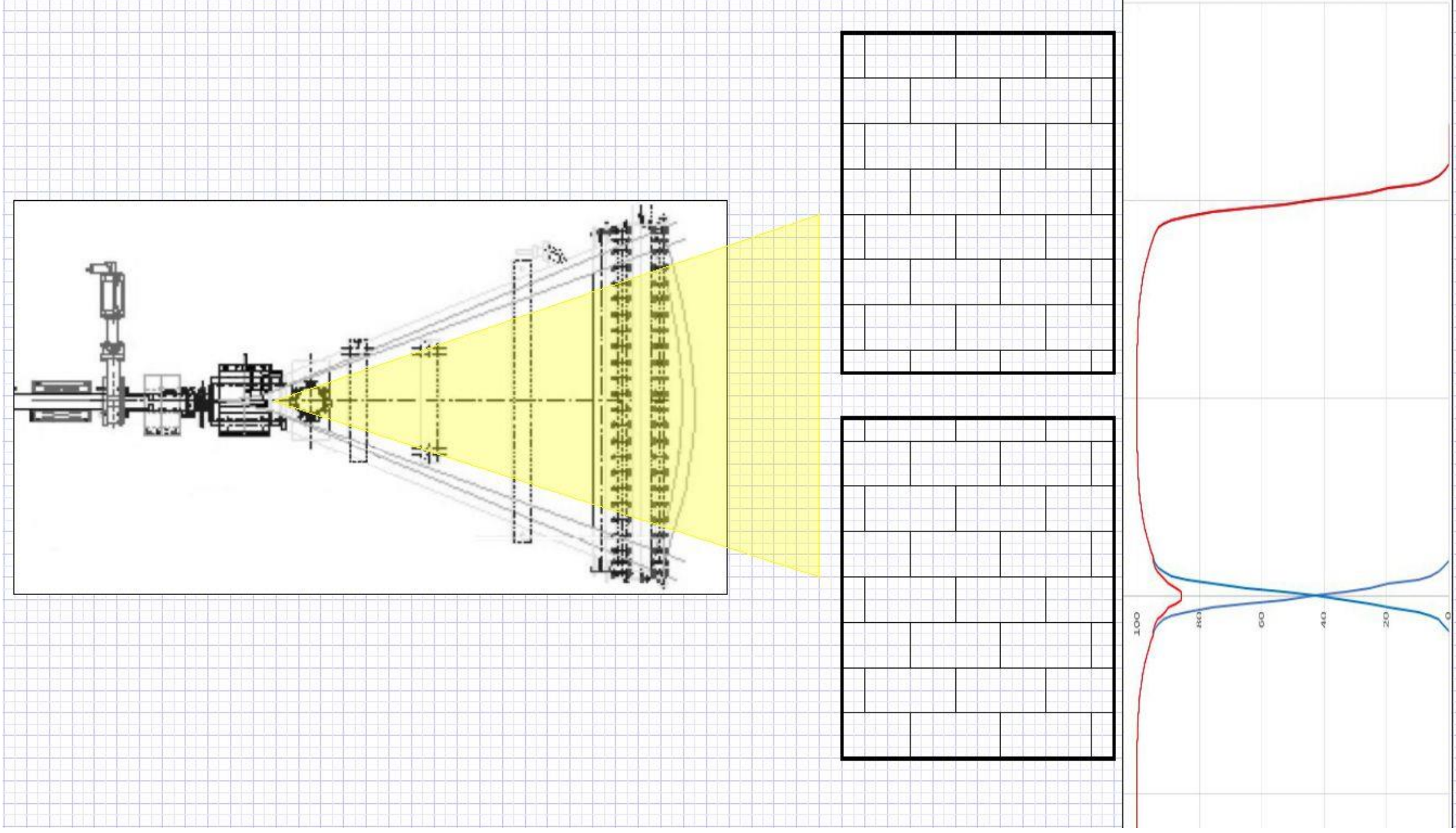


\section{Capacity considerations (Courtesy: T.Kroc FNAL)}

- $1 \mathrm{Mci}=3.7 \times 10^{16}$ decays/second

- Total energy released $-2.505 \mathrm{MeV} /$ decay

- $15 \mathrm{~kW}$

- Typical irradiation bunker - 30-60 kW of "beam" power

- Electron beam machines can provide this easily

-X-ray must overcome inefficiency of Bremsstrahlung process

- $200-400 \mathrm{~kW}$ of electron beam power

- Then must include efficiency of electron beam production 


\section{Capacity considerations}

- Gamma (typical capacity is $2-4 \mathrm{MCi}$ )

- $\sim 10 \mathrm{kGy} / \mathrm{hr}$

-3.4 m³/h/MCi @ 25 kGy

- Electron Beam

- $20 \mathrm{MGy} / \mathrm{hr}$

- X-ray

- $\sim 60 \mathrm{kGy} / \mathrm{hr}$

- 2.8 m³/h/100 kW @ 25 kGy (including target losses)

$1 \mathrm{MCi}$ gamma $\approx 120 \mathrm{~kW}$ X-ray <- Need high power e-beam sources to match current and emerging neeeds. 


\section{Wastewater Multitool that Treats Contaminant X in Water}

\section{Military}

- Explosives, PCBs, TCE

- Treat military PFCs problem today...

- 200 military facilities

- \$Bs of emerging contaminants

\section{Energy Production}

- Point source contamination

- Total water re-use

- 10,000 large processing facilities

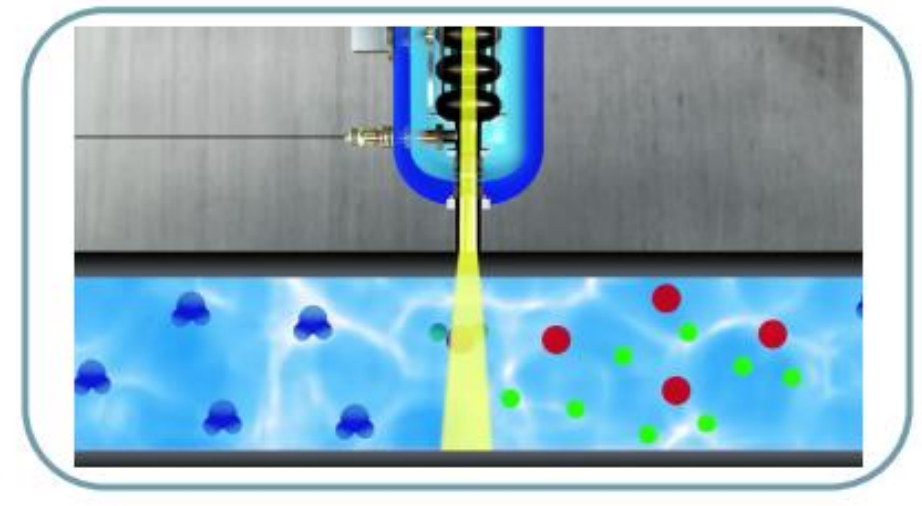

\section{Municipal}

- Treat PFCs, pharmaceuticals, bacteria/viruses

- Quick to respond to EPA regulation

- > 16,500 public treatment facilities

\section{Industrial}

- Chemical manufacturing, textiles, medical

- Point source contamination

- Reduce/Re-use

\section{Food \& Beverage}

- Reduce/Re-use

- Condition incoming water

- Clean in place process 


\section{Electron Beam Treatment of Water}

$\mathrm{H}_{2} \mathrm{O} \underset{\text { Electron }}{\text { Beam }} \longrightarrow \mathrm{OH}-, \mathrm{H}+, \mathrm{e}_{-\mathrm{aq}}, \mathrm{H}_{2}, \mathrm{H}_{2} \mathrm{O}_{2}$

- Increases dewaterability

- Removal of toxic chemicals not removed in conventional domestic water treatment: Pharmaceuticals, Agricultural run off, Fuel additives (MTBE), PCBs, PFAS/PFOA perfluorinated compounds

- Reduction in pathogens

- No toxic residuals (no secondary waste generation)

- Increased phosphorus recovery

Conventional E-Beam technology shown to remove many contaminants in water but

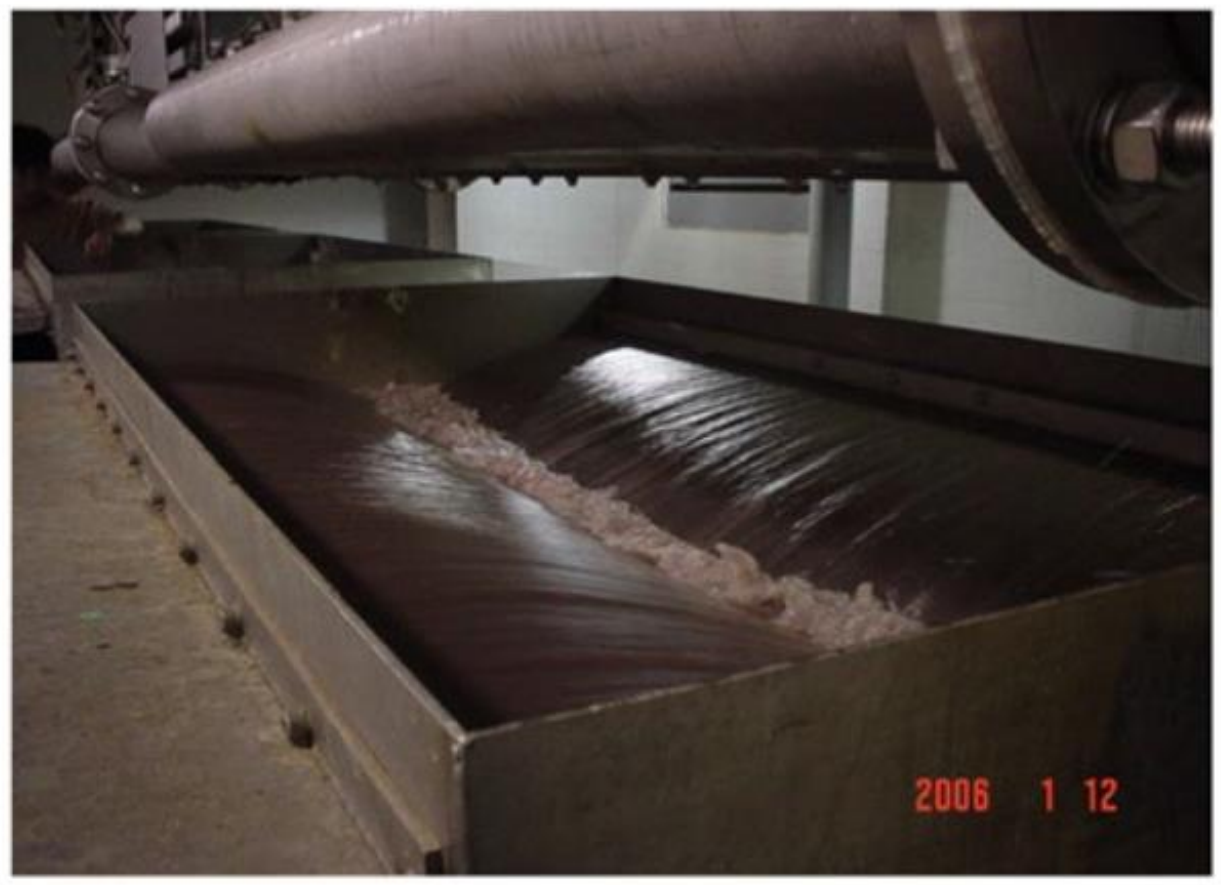

Picture of conventional e-beam

- Low Throughput

- Energy Intensive

- Large Nonportable Footprint 
Fresh from the press....

CWN heàlth

\section{Makeup may contain potentially toxic chemicals called PFAS, study finds}

By Sandee LaMotte, CNN

Updated 7:46 PM ET, Tue June 15, 2021

(CNN) - The "No PFAS in Cosmetics Act" was introduced in the US House and

Senate on Tuesday, following the release of a new study that found high levels of

a marker for toxic PFAS substances in 52\% of 231 makeup products purchased 


\section{E-Beam Treatment of PFASs}

- Per- and polyfluoroalkyl substances (PFAS) are a group of man-made chemicals including PFOA and PFOS.

- They are found in the blood serum of $99 \%$ of people in the world wit no conventional treatment technique.

- E-beam can breakdown PFASs to salts, $\mathrm{CO} 2$ and water. Currently investigating large scale application.
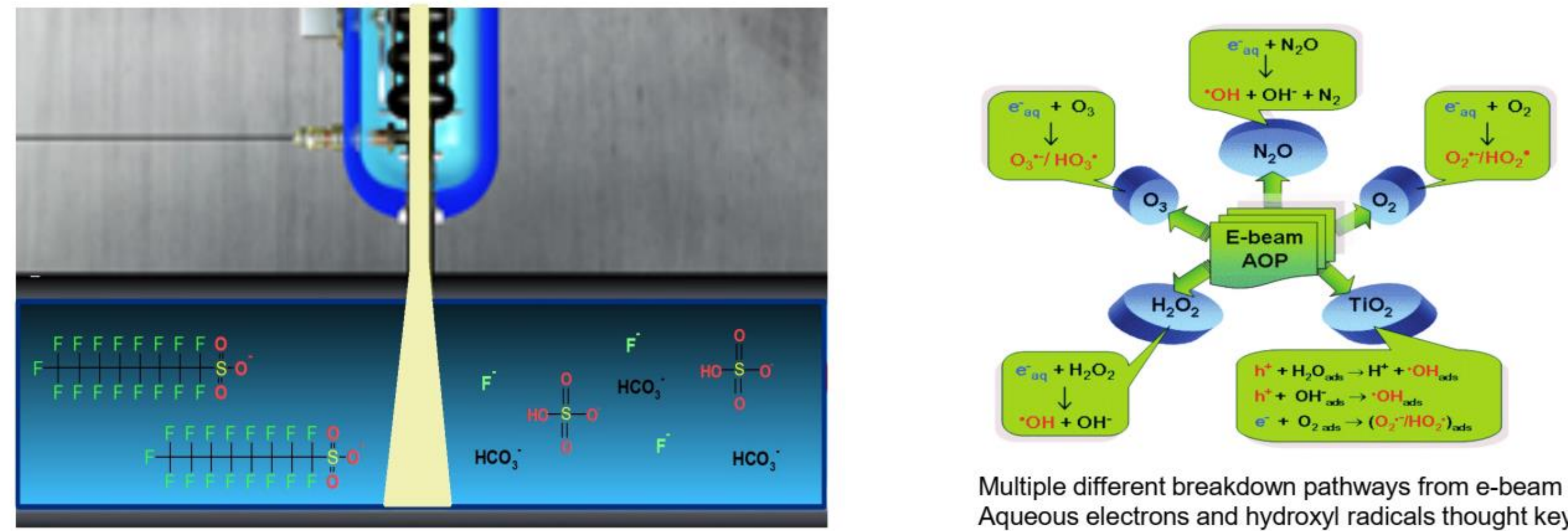

Multiple different breakdown pathways from e-beam . Aqueous electrons and hydroxyl radicals thought key for PFAS destruction. 


\section{Challenges on the 'roads ahead':}

- $94 \%$ of road in the US are asphalt.

- $>50 \mathrm{~B} \$$ /year in renewing asphalt pavement

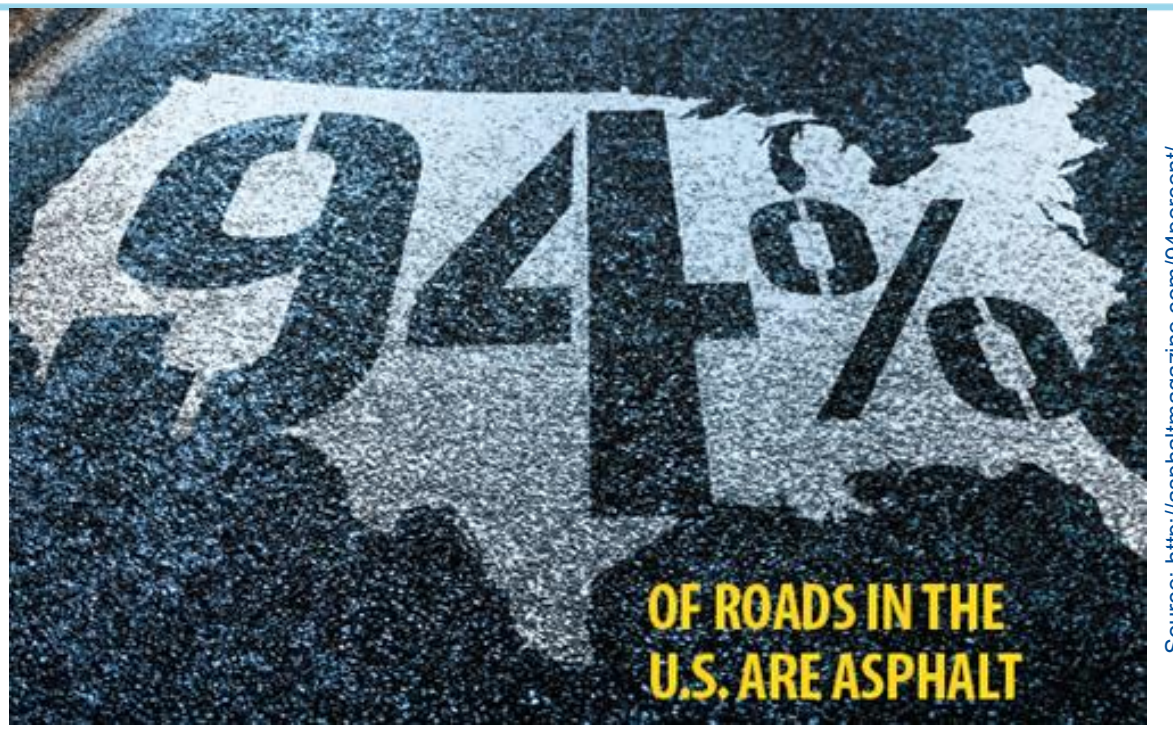

- Asphalt pavement damage is largely due to cracks of the bitumen binder under heavy load. Oxidation, water freezing and thawing in cracks all create a continuous and expensive cycle of renewal, exacerbated in colder climates

- Despite attempts to improve asphalt pavement, the materials and fabrication method have changed little for several years. 


\section{Leverage accelerator technology to extend pavement lifetime}

- $\quad$ IARC at Fermilab has partnered with U.S. Army Corps of Engineers ERDC via an interagency agreement on a R\&D project to extend the lifetime of pavement using modern accelerator technology

- Our technology will improve the strength, toughness and the service of the paved surfaces.

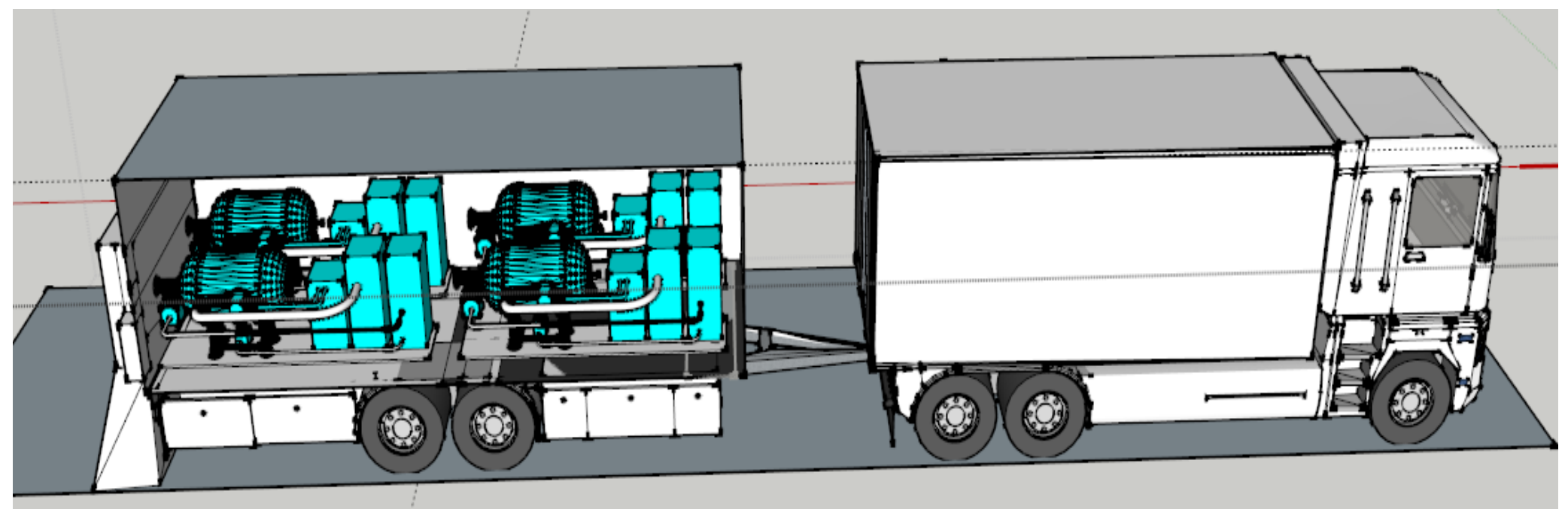




\section{Many emerging areas that SRF accelerators can add value}

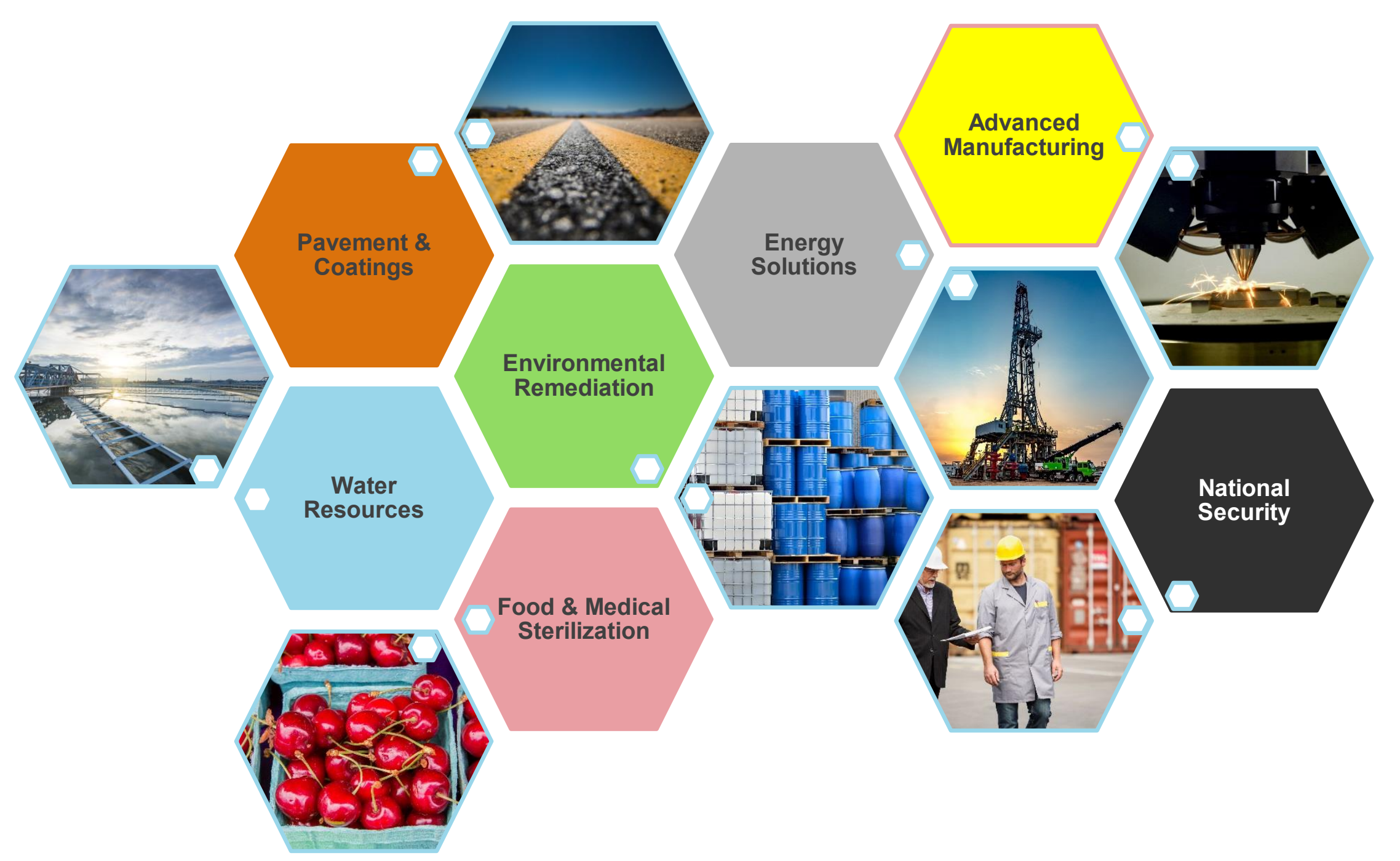




\section{Conclusions}

- Lot of activity is on-going with a goal to make an entirely new class of industrial SRF-based electron accelerators that use no liquid cryogens

- Mobile, high energy, high power, high efficient electron accelerators can enable a variety of entirely new industrial applications

- Several applications may have enormous market potential

- If you are an interested in working with Fermilab Tech or from Fermilab, talk to me for opportunities!

- If interested in commercialization of our tech, we have resident experts at IARC and OPTT. I will be happy to put you in touch.

- Questions?????? 